\title{
Claves teóricas para una nueva visión de la recepción mediática desde la experiencia migratoria
}

Recibido: 28 de enero de 2011

Aceptado: 22 julio de 2011

Publicado: 31 de octubre de 2011
Luis Concepción Sepúlveda LuisGilbertoCS@blanquerna.url.edu Alfons Medina Cambrón alfonsomc@blanquerna.url.edu (Universidad Ramon Llull - España)

Resumen: Este artículo ofrece una nueva visión para la investigación en comunicación de la recepción mediática a través de la relación que se establece entre la sociedad de acogida y la diáspora. La recepción mediática de la inmigración, además, es uno de los campos que cuenta con menos bibliografía en España. Mostraremos, por tanto, la importancia de generar otras líneas de investigación en comunicación que se acerquen al estudio de la nueva realidad que se está formando, actualmente, en nuestras sociedades. Las migraciones ponen en cuestión no sólo la visión tradicional de la experiencia mediática que venía haciendo la investigación española a partir de un público nacional y sedentario, sino que también replantea el papel del medio de comunicación con la aparición de los nuevos medios dirigidos a los inmigrantes.

Palabras clave: Recepción, inmigración, medios de comunicación, "Mundo de la vida" [Lebenswelt], investigación en comunicación, metodología.

Abstract: This article shows a new vision of Media Consumption for Communication Research through the relation between a host society and Diaspora. Immigrant's Media Consumption is already one of the fields with less bibliography in Spain. Therefore, we show the importance of generating other lines of Research in Communication that approach the study of the new reality that is forming at present, in our society. Migrations put in question not only the traditional vision of the media experience from Spanish Research with a national and sedentary public, but also it reexamines the role of Mass Media aimed to immigrants. 
Key words: Reception, Immigration, Mass Media, "Lifeworld”, Communication Research, Methodology.

\section{Introducción ${ }^{1}$}

Desde finales de la década del noventa, España ha asumido un nuevo papel en relación a la emigración. De ser una sociedad emisora de emigración, se ha transformado en una sociedad receptora de dicha emigración. El trabajo que aquí presentamos, aunque sin obviar las relaciones existentes, no tratará de las consecuencias sociales, políticas o económicas de este fenómeno. Nos ocuparemos de la relación que se establece entre la sociedad de acogida y las personas que han llevado a cabo el proceso migratorio, pero centrándonos en la elaboración de nuevos marcos teóricos que sean más adecuados para comprender las nuevas estructuras sociales y su relación con los medios. Mostraremos, por tanto, la importancia de generar otras líneas de investigación en comunicación que se acerquen al estudio de la nueva realidad que se está formando, actualmente, en nuestras sociedades.

Para contextualizar y ejemplificar de manera diáfana este fenómeno, que modifica la estructura social de España y también de Cataluña, ofreceremos algunos datos. Las cifras oficiales del Instituto de Estadística de Cataluña (2000) muestran la presencia de 181590 ciudadanos extranjeros. Este dato representa el 2.9\% de la población catalana. En el año 2010, en Cataluña, la población extranjera es del 15.95\% (1 198538 personas), de los cuales un 30\% son de otros Estados de la Unión Europea o del resto de Europa; y un $70 \%$ de África, América y Asia. Ya en 2008, este porcentaje había alcanzado el 15\% de la población catalana. En los últimos tres años, con las medidas restrictivas por parte de los diferentes estados de la Unión Europea (para frenar la llegada de personas provenientes de otros Estados) y con el inicio de la crisis económica mundial, el crecimiento ha sido muy tenue (IDESCAT, 2010).

Esta nueva estructura social comporta, más si cabe, la necesidad de ofrecer un giro a la investigación en comunicación en relación al tema planteado. En dicho ámbito, las teorías que han predominado durante décadas, y como consecuencia de esto también en la investigación, han sido las que han tenido como objeto principal el estudio de la influencia de los medios de comunicación sobre las personas, los públicos o las audiencias. Este modelo lineal, basado en el paradigma de Lasswell (Moragas, 1993), ha fundamentado la investigación predominante a lo largo de décadas, y aunque superado y matizado por teorías posteriores, la visión y el estudio de las influencias sigue teniendo presencia teórica y también, evidentemente, mediática (Medina, 2010).

1.- Este artículo se basa, en primer lugar, en la investigación y los diferentes resultados (en forma de publicaciones) que realizamos un grupo de investigadores de la Facultad de Ciencias de la Comunicación Blanquerna (Universitat Ramon Llull), entre los años 2008 y 2009, sobre los usos y actitudes de los emigrantes ante los medios de comunicación (Altarriba M. et álii. Usos i actituds dels immigrants davant dels mitjans de comunicació. Barcelona: Consell de l'Audiovisual de Catalunya, CAC); y, posteriormente, de manera fundamental, a partir del desarrollo de la tesis doctoral (que se encuentra en proceso de elaboración) de uno de los autores del presente artículo. 
En este sentido, hasta hace relativamente pocos años, la investigación comunicativa se centraba únicamente en la imagen y, sobre todo, en los estereotipos de los emigrantes en los medios de comunicación tradicionales. Hoy el estudio de las relaciones que se dan entre la comunicación y la inmigración sigue circunscribiéndose, preferentemente, al papel de los medios de comunicación de masas en la representación de la emigración. Es la línea de exploración que marca la tendencia hasta la actualidad, lo que deja prácticamente arrinconadas, por ejemplo, áreas como la vertiente interpersonal de la comunicación y el análisis comunicacional de las redes de inmigrantes, que apenas se vislumbran en nuestro contexto geográfico ${ }^{2}$.

De esta forma, la investigación universitaria se preocupó, en un principio, por una cobertura mediática del nuevo fenómeno que crecía exponencialmente. El análisis de contenido de este tipo de noticias, centradas en su mayoría en la llegada de subsaharianos y marroquíes a las costas españolas por medio de pateras ("embarcaciones rudimentarias"), intentaba arrojar una luz más sosegada ante la escalada informativa de signo negativo. Así, desde mediados del noventa, los trabajos más ambiciosos han puesto en marcha la deconstrucción del discurso periodístico hacia niveles de lectura más profundos para evidenciar los significados y las estrategias textuales de mayor calado ${ }^{3}$. Son trabajos que han puesto de manifiesto que la asimetría social, jurídica y económica de los migrantes y de las minorías tiene su traducción textual en el discurso informativo.

Con el paso de los años se produce un avance hacia una sociedad más intercultural a medida que el peso demográfico de la inmigración es más significativo. Sin embargo, el relato periodístico no acierta a ver el carácter noticioso de una estructura social que estaba cambiando para siempre con nuevos colectivos que habían dejado de ser un fenómeno externo y eventual para formar parte del entorno de manera estable. El ámbito académico también se quedó a la zaga en esta nueva fase, perdiendo la oportunidad de analizar desde el ángulo de la comunicología los cambios más importantes de una transición hacia un nuevo paisaje social.

Durante la primera década del siglo XXI, España se ha convertido en uno de los países con más número y crecimiento porcentual de inmigrantes de la Unión Europea (Vasileva, 2010), ofreciendo un nicho de estudio único. Sólo unos pocos académicos y grupos de investigación especializados en comunicación siguen los pasos de una de las transformaciones sociales más grandes de la historia moderna española. Se inicia, así, una paulatina evolución de la inmigración como objeto de estudio que deja atrás el interés inicial por el análisis de contenido de la prensa de pago, para dar paso a una lenta apertura hacia la recepción mediática de la inmigración como una nueva audiencia. Y aunque la producción científica de la recepción mediática (sea sobre el impacto de las noticias de

2.- Dos de las aproximaciones más sólidas al estudio de la inmigración desde un concepto amplio de la comunicación humana son las tesis doctorales de González Escudero (1999) y de Rizo García (2004) presentadas en la Universidad Autónoma de Barcelona.

3.- Hay que reconocer que la aplicación de las técnicas cualitativas de algunas corrientes como los estudios de género, el análisis crítico del discurso, la comunicación intercultural, la semiótica narrativa (inspirada en Greimas), la criminología crítica, la "teoría del encuadre" y la agenda setting, conforman un abanico amplio y selecto con interesantes aportaciones en el estudio de las representaciones de la diáspora. 
inmigración entre los nacionales o desde los usos sociales que los inmigrantes hacen de los medios) sigue creciendo a un ritmo pausado, la hegemonía continúa estando en manos del análisis textual de las noticias.

La mayoría de trabajos focalizados en la recepción mediática de la inmigración carecen, de un lado, de propuestas teóricas y metodológicas que permitan superar una mirada excesivamente institucional (a menudo deudora de las entidades que subvencionan y rigen las investigaciones); y del otro, redundan en un sesgo etnocéntrico. Y es que los investigadores no son suficientemente conscientes de la carga cultural de la sociedad mayoritaria en la que viven. En realidad, son muy escasas las incursiones críticas que aprehenden la inmigración fuera de las teorías asimilacionistas de los países desarrollados.

Al no contar con marcos teóricos bien nutridos, de acuerdo con los objetivos de una investigación rigurosa, hay un estrecho margen de maniobra para interpretaciones de largo recorrido del material registrado. Como recuerda el colectivo IOÉ (2010: 95) al hablar de los trabajos sobre inmigración, "aquí cuentan las opciones metodológicas y, más aún, las concepciones teóricas de fondo", así como un posicionamiento claro sobre lo social.

\section{Objetivos}

2.1. Este artículo se marca como objetivo impulsar un marco teórico y metodológico que rompa con una mirada científica que estudia la recepción mediática de la migración desde el punto de vista exclusivo de la sociedad de acogida y desde la utilidad del medio de comunicación para la integración del inmigrante. El estudio de la recepción mediática tiene que liberarse de esta orientación marcadamente instrumental con el objeto de estudio (la inmigración) y profundamente funcionalista en la concepción utilitarista del medio de comunicación para la asimilación del inmigrante en la sociedad receptora. La ruptura con la tendencia a plantear metodologías (que no toman en cuenta que han sido concebidas en el seno de una sociedad mayoritaria) abre nuevas vías para la generación de un conocimiento científico más libre y desprejuiciado en el estudio de la experiencia mediática.

Los investigadores tampoco son ajenos a la influencia del debate político sobre la integración de los inmigrantes: un discurso amplificado por los medios que se acentúa durante las campañas electorales. Las actitudes y argumentos esgrimidos en la instrumentalización política de la inmigración son empleados en la contienda como un arma arrojadiza. Sin duda, su influjo se filtra a todos los niveles de la sociedad como una gran corriente de opinión. La inmigración emerge así de forma más o menos consciente como un objeto de estudio al que hay que supervisar porque puede poner en peligro la cohesión social.

Estamos convencidos de que hay que dejar atrás a la inmigración como un fenómeno social externo a la sociedad de acogida para entenderla como una comunidad de comunicación (Habermas, 1999b: 560) que participa activamente como ciudadanos de pleno derecho.

2.2. Queremos contribuir a la apertura del estudio de la inmigración desde las Ciencias de la Comunicación, más allá del análisis de contenido de la prensa generalista. Pretendemos 
aproximarnos a la apropiación mediática de la diáspora desde los significados y los procedimientos de interpretación de las propias minorías. De esta forma, podemos conseguir una mayor comprensión de la relación que se establece entre los medios especialmente dirigidos a la inmigración y los inmigrantes, así como el papel real de la experiencia mediática en sus biografías.

2.3. Dotar de un instrumental teórico sólido y bien estructurado que permita lecturas creativas y verosímiles de la recepción mediática de la inmigración en conexión con las diferentes áreas de la vida cotidiana. Queremos reflejar su protagonismo en la singularidad del sujeto, en las relaciones sociales y en la cultura. Se trata, en definitiva, de partir de un concepto elaborado de receptividad mediática que nos deje superar la óptica descriptiva en la que se inscribe la mayor parte de trabajos. Si bien lo que abunda es la descripción objetiva de las elecciones mediáticas de los sujetos de estudio, nosotros queremos entrar a interpretarlas y ponerlas en relación con sus respectivos marcos de influencia: el sistema, el individuo, la sociedad y la cultura.

\section{Metodología}

Existe cierta confusión terminológica en relación a la definición del concepto "metodología". Podemos hacer referencia a este concepto en dos sentidos. En primer lugar, la metodología nos indica el modelo teórico elaborado y el paradigma en el que se sitúa una determinada conceptualización. Cuando hablamos de metodología, no nos referimos exclusivamente a las técnicas de recogida de datos, sino también a la conceptualización, la exploración y una rigurosa adecuación de la investigación entendida como un proceso global. La relación de técnicas utilizadas, el trabajo de campo propiamente dicho, se encuentra en proceso de elaboración.

No obstante, lo que sí se presenta en este artículo es el andamiaje conceptual y teórico que será fundamental para su elección y elaboración. No podemos confundir, por tanto, la metodología con los métodos (en plural) de investigación que utilizaremos. Para cualquier explicación sobre la metodología tenemos que intentar conseguir un equilibrio que tenga en cuenta la investigación desde una visión teórica global y una explicación detallada de las técnicas que utilizaremos (Medina, 2010: 149).

La investigación que se está llevando a cabo se sitúa dentro de un paradigma sociocrítico y comunicativo. Es decir que, entre otros factores, es necesario tener en cuenta la influencia del contexto cultural, social y relacional, cuando se interpreta la realidad investigada. Nuestro marco teórico se nutre de las propuestas intersubjetivas y de perspectivas comprensivistas y de los teóricos de la construcción social de la realidad, que justifican un acercamiento cualitativo a los grupos de estudio y que servirán también para contextualizar los medios de comunicación como creadores de realidad. En este sentido, uno de los elementos centrales de todo el proceso de búsqueda es el acercamiento a los colectivos inmigrantes para tener sus opiniones sobre el tema. En definitiva, creemos en la importancia de la interacción con los sujetos implicados. 


\section{Hipótesis de trabajo}

La hipótesis general de partida de la investigación en curso, y cuyo desarrollo y avances mostramos en este artículo, nace de la voluntad de hacer una lectura de la recepción mediática de mayor alcance. La receptividad está, así, íntimamente vinculada a tres esferas diferentes e interrelacionadas: personalidad, sociedad y cultura. Esto quiere decir que la recepción está en relación con el desarrollo personal de los inmigrantes de una forma no investigada hasta la fecha; permite trascender la situación personal en conexión con el mundo que rodea a la experiencia migratoria; activa o refuerza mecanismos de solidaridad entre inmigrantes; impulsa espacios de encuentro y comunicación; y establece canales más sólidos para la articulación de la acción cívica, política y cultural dentro del propio grupo.

\section{Renovación de la terminología}

Antes de empezar a desgranar nuestro programa teórico, explicaremos por qué hemos escogido algunos términos para referirnos a la inmigración. En la búsqueda de palabras (que no estén tan viciadas de un significado negativo ni tengan orientaciones semánticas tan marcadas) pondremos especial cuidado en los términos que emplearemos para evitar al máximo una mirada parcial del fenómeno migratorio. Como recuerda Gadamer (2002: 7), el lenguaje forma parte de un mundo preformado y convencional dentro de los límites de un territorio. En este punto, sabemos que parte de la imagen negativa que se proyecta de la diáspora tiene que ver con los recursos simbólicos que se emplean en las narraciones sociales. Además, dada su importancia para la cohesión social, su protagonismo en la actualidad informativa y en los debates públicos, la elección léxica rigurosa debería ser un imperativo categórico en la literatura científica.

Aspectos de actualidad (tales como la movilidad creciente de las personas inmigrantes a través de las cadenas migratorias de familiares y amigos; o los circuitos transnacionales creados por la inmigración; la capacidad de las personas para vivir física y tecnológicamente entre dos o más países, gracias al progreso y abaratamiento de los medios de transportes y de las tecnologías de la información y comunicación) son factores de una nueva inmigración desconocida hasta el momento. La intensidad con la que conceptos como el transnacionalismo han entrado en juego pone al descubierto el desfase de palabras que han perdido la capacidad de transmitir con fidelidad esta nueva experiencia migratoria. Porque “inmigración” es un término más estático que designa en general la entrada de personas en un país, pero no el dinamismo físico y social de los contactos entre individuos en diferentes países.

El término "diáspora", en cambio, es más adecuado porque nace para expresar la diseminación de pueblos (como el judío) que hicieron del éxodo su razón de ser. Su campo semántico es más flexible para reflejar mejor que otra palabra la diseminación continuada de determinados pueblos inmigrantes alrededor del mundo. La definición que trae a colación la palabra "inmigración", por contra, tiene demasiadas hipotecas puestas tras los barrotes de los Estados nación. No es de extrañar que resulten insuficientes para referirnos 
a un movimiento poblacional que no empieza ni acaba en el país de llegada, por lo que no se puede estudiar descontextualizado, dentro de los confines de un Estado nación, sino que hunde sus raíces en la transnacionalidad como una forma de vida.

Y si la diáspora trae ante nosotros la dimensión transnacional que ha adquirido la inmigración (como resultado de los procesos de mundialización de los últimos años) otras expresiones como "minorías étnicas" inciden más bien en la relación de fuerzas entre la sociedad mayoritaria y la diáspora, en clara desventaja. También acentúa la voluntad de permanencia de colectivos que se conciben a sí mismos como un grupo diferenciado de ciudadanos con una serie de particularidades.

El cruce de estos dos conceptos ("diáspora" y "minorías étnicas") nos va a aportar un enfoque más amplio, profundo y real de este fenómeno social.

\section{Marco teórico para una nueva teoría de la recepción mediática}

\subsection{De camino a Habermas}

El marco teórico de la investigación que se está llevando a cabo se inspira en la "teoría crítica comunicativa” de Jürgen Habermas (1994a, 1994b, 1996, 1999a, 1999b, 1999c), quien desdobla la sociedad en dos grandes "esferas", tan aparentemente opuestas como complementarias e interrelacionadas: el denominado "mundo de la vida" y el "sistema". La incorporación de estas categorías analíticas en el estudio de la recepción permite desarrollar una interesante guía de lectura de la interacción entre las minorías étnicas y los medios especializados en ellas.

"Mundo de la vida" y "sistema" se aproximan a la apropiación mediática desde un marco social, cultural e individual más amplio, que al mismo tiempo refleja las dinámicas relacionales entre la cultura, la sociedad y la personalidad. Así, podemos acercarnos a la actividad receptora sin arrancarla de la trama en la que toma forma, para relacionarla con sus procesos sociales, culturales y de la personalidad de la vida cotidiana. La actividad receptora está indisolublemente entretejida en la vida cotidiana, por lo que estudiar la recepción descontextualizada de sus dimensiones sería como si en zoología, por ejemplo, se quisiera aislar a un animal recientemente descubierto para analizarlo en un laboratorio, fuera de su medio natural.

En definitiva, la interpretación habermasiana de esta sociedad dual en la que viven y colisionan los intereses del sistema con el ecosistema frágil del mundo de la vida puede dotar a nuestro análisis de un mayor alcance. Nos garantiza interpretaciones no fragmentadas del papel que ocupa la recepción en el interior de una comunidad "diaspórica".

\subsection{Mundo de la vida o Lebenswelt}

El "mundo de la vida" es el eje de nuestra propuesta teórica. Se trata de un concepto que a lo largo del siglo XX ha experimentado un gran desarrollo teórico y que puede ser capital para la investigación científica en Ciencias de la Comunicación. Si se hilvana su evolución 
a través de las aportaciones de pensadores tan importantes como Edmund Husserl, Alfred Schütz y Jürgen Habermas, veremos que es una pieza clave para triangular, por un lado, la reproducción cultural, la integración social y la socialización; y por otro, para poner en conexión estos elementos con el sistema.

Esta multidimensionalidad también le confiere cierta capacidad para captar la movilidad de procesos que se manifiestan en la vida cotidiana y que atraviesan varias de estas esferas. "Mundo de la vida" también es un término difícil de glosar con una definición que esboce todas sus posibilidades teóricas. Sin embargo, se puede adelantar brevemente que es una forma de saber global y cotidiano que no está reglada o formalizada según los criterios de la cultura moderna. Asimismo, es un espacio de encuentro en el que es posible la comunicación sobre la base de conocimientos compartidos inspirados o tomados de la tradición (Habermas, 1999b).

Durante el primer cuarto de siglo XX, Husserl (1991: 3) diagnostica en La crisis de las ciencias europeas y la fenomenología trascendental que las ciencias están en crisis porque el modo en que se autoproponen objetivos y tareas, generan, en consecuencia, una metodología que se ha vuelto problemática. La entrada en escena de las matemáticas modernas de Galileo, encumbradas siglos más tarde por la corriente positivista, es la causa por la que la ciencia toma las "cosas del mundo" para transformarlas en formas fijas y universales para el interés teórico (Husserl, 1991: 26-27).

En esta forma de pensar, sin embargo, no se tiene en cuenta que las entidades del mundo son objetos culturales que forman parte de una totalidad unitaria con un sentido preestablecido para una comunidad de personas definida como "nosotros". Los científicos, critica Husserl, elaboran sus teorías sin caer en la cuenta que los elementos tienen una prehistoria social y forman parte de un legado de una vida intuitiva y precientífica que ya ha clasificado todas las cosas (Acebes, 2000).

Desde la filosofía, Husserl denuncia que las ciencias (primero las ciencias naturales influidas por las matemáticas galileanas y luego, por extensión, las ciencias sociales) se han cimentado sobre el subsuelo de las autoevidencias del "mundo de la vida" sin que las ciencias se hayan preguntado científicamente por la naturaleza de lo mundano (Husserl, 1991: 130-138). El mencionado autor ponía al descubierto, de esta manera, la supuesta objetividad científica construida socialmente en base a un conocimiento compartido por los miembros de una comunidad. La introducción de este, a priori, lo que viene a ser la primera forma del "mundo de la vida" en su evolución teórica, inaugura un nuevo comienzo para una ciencia que debía ser más rigurosa y que debe poner entre paréntesis las pretendidas certezas y obviedades del "mundo de la vida" como fenómenos a estudiar.

La recepción de "mundo de la vida" en Schütz lanza el concepto hacia su redimensión sociológica, lo que nos ofrece la base para un conocimiento significativo del trasfondo en el que actúan las personas. Siguiendo a Habermas, se ha complementado esta idea de "mundo de la vida" con la intersubjetividad y el carácter dinámico y transformador que le dan los procesos comunicativos a partir de las interacciones de las personas que viven, actúan y piensan en dicho entorno, así como el carácter dual del que carecen los acercamientos de tipo fenomenológico ("mundo de la vida" y "sistema"). 
Habermas redondea un término que seguía anclado en una definición culturalista heredada de la tradición fenomenológica de Husserl y Schütz ${ }^{4}$. El Lebenswelt llega a Habermas como convicciones culturales que proveen patrones de interpretación, valoración y expresión, aceptados socialmente y que permanecen incuestionados (Habermas, 1999b: 315). No sólo incorpora estos presupuestos de fondo con los que el ser humano enfrenta sus grandes áreas de opacidad de la vida, sino que los reinterpreta a la luz de una nueva teoría de la comunicación. Da así una mayor movilidad a un término demasiado ligado a la conciencia individual (Rodríguez Salazar, 1996) y establece conexiones sólidas con otros procesos de la vida cotidiana, como la reproducción cultural, la integración social y la socialización. Estos procesos se engloban, en el mismo orden, en las categorías "cultura", "sociedad" e "individuo" (Habermas, 1994a: 497-498; 1994b: 38; 1999b: 196). Su articulación simbólica integra, de esta forma, tres grandes componentes estructurales que se influyen recíprocamente en un sistema de relaciones de interdependencia (Concepción, 2009:110).

\subsection{Aplicaciones del marco teórico}

Las aplicaciones de esta teoría en el estudio de las apropiaciones mediáticas son muy diversas. Por esta razón, hemos decidido agruparlas en tres grandes conjuntos que inciden en diferentes niveles o grados de nuestra investigación: desde el más general (el enfoque global del estudio) a más concretos, como el escenario en el que tiene lugar la recepción, la especificidad de la audiencia migrante y la propia recepción.

\subsubsection{Un enfoque global al estudio de campo}

En primera instancia, la aplicación del modelo crítico de sociedad que propone Habermas permite relacionar dos niveles de análisis de un estudio de campo. Establecemos una dimensión microsocial que viene dada por la experiencia cotidiana del "mundo de la vida" de la audiencia y una dimensión macrosocial, determinada por formas más objetivas de organización social del sistema (Radl, 1998: 106). De esta manera, damos con un concepto de apropiación mediática que incluye contradicciones del capitalismo avanzado ${ }^{5}$, como la legislación de extranjería y la economía de mercado; y un Lebenswelt desarraigado de sus referentes territoriales y culturales, que se manifiesta de forma subjetiva, pero también de manera colectiva.

Así las cosas, esta teoría dual de la sociedad confiere a nuestro estudio un enfoque global porque centra su atención en la relación de complementariedad entre los niveles microsocial y macrosocial y la sitúa por encima de otros temas. Con estas pautas, el investigador social

4.- La suma del “interaccionismo simbólico", de Georg H. Mead, y la categoría filosófica del "mundo de la vida" de Husserl y Schütz, permite a Habermas plantear la acción comunicativa con bases firmes (Concepción, 2009: 110).

5.- El sistema es el resultado directo de dos dinámicas, el capitalismo y el Estado, que a lo largo de la historia han incrementado su complejidad hasta el punto de colonizar el propio "mundo de la vida" ("colonización sistémica del mundo de la vida"). Dicha complejidad a partir de un proceso creciente de racionalización, apuntado ya por Weber (1998), ha colocado en el lugar de las decisiones, en un espacio privilegiado, el dinero y el poder, dos elementos que se derivan del capitalismo y del Estado. Actualmente, el despotismo del dinero y del poder ha creado nuevos niveles de integración social que no dependen de la cultura y el lenguaje dentro del "mundo de la vida". La coexistencia de ámbitos opuestos marca las contradicciones y parámetros con los cuales tienen que vivir las personas. 
se abre a la dialéctica entre los aspectos individuales del sujeto y los elementos de la estructura social (Radl, 1998: 114).

Dicho modelo de sociedad aporta también una visión pragmática de un lado, que analiza los usos sociales desde las necesidades que impone la vida cotidiana; y una visión contextual del otro, porque introduce las condiciones socioestructurales del mundo de la vida y del sistema desde una vertiente más teórica.

\subsubsection{Elementos socioculturales de la apropiación mediática}

La categoría "mundo de la vida" puede ser operativa para analizar un consumo mediático desde un marco sociocultural más dinámico y complejo donde los sujetos no son estudiados como actores solitarios. Ahora nos interesa dibujar el escenario en el que interaccionan los diferentes elementos de la sociedad que inciden en las apropiaciones mediáticas.

Figura 1: Elementos socioculturales de la apropiación mediática.
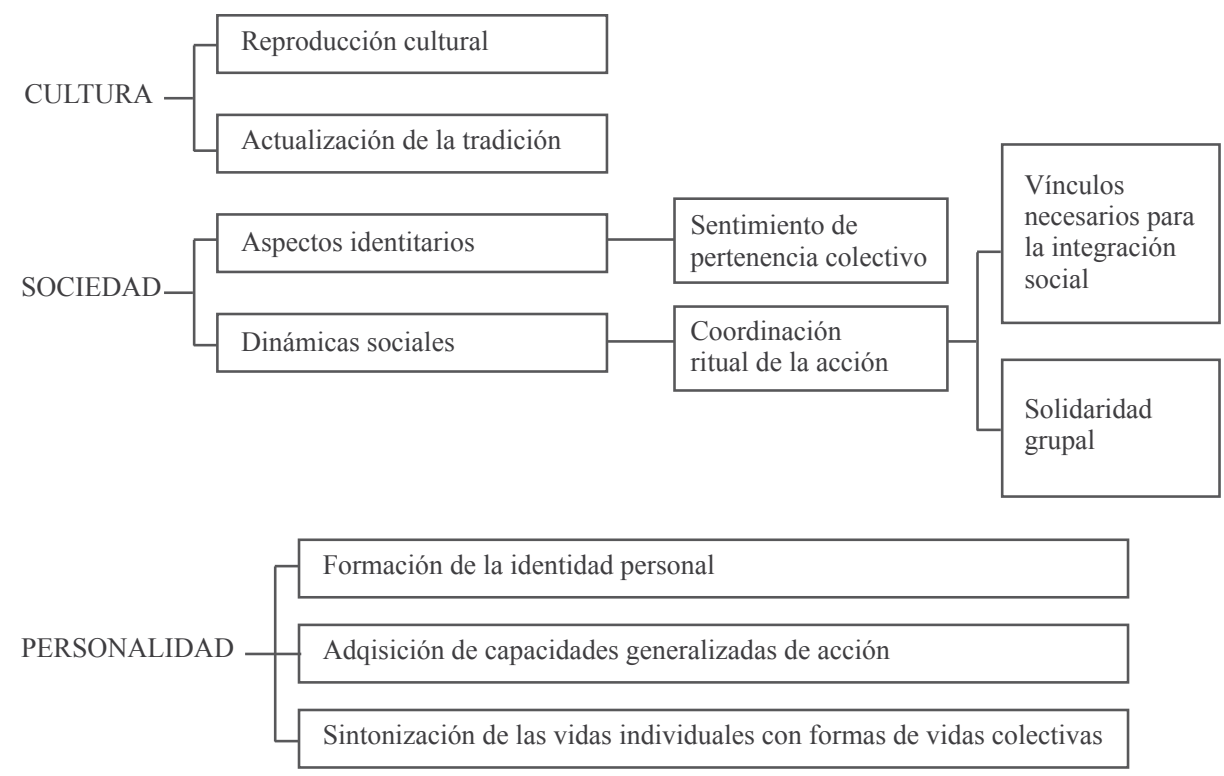

Fuente: Elaboración propia a partir de Habermas (1999b).

Habermas ofrece un campo de análisis estructurado y definido a través de tres coordenadas muy claras: "sociedad", "cultura" y "personalidad” (Rodríguez Salazar, 1996). El juego de mutuas influencias entre estos componentes explica las dinámicas internas de la realidad social. De esta manera, se obtiene el guión de los movimientos socioculturales, así como una descripción de los papeles que interpretan los actores que intervienen. 
$\mathrm{Si}$ atendemos al esquema (figura 1), veremos que nuestro modelo teórico tiene una dimensión social que engloba e interpreta los aspectos más intangibles y anímicos de los movimientos identitarios, como el sentimiento de pertenencia grupal. Pero tampoco escapan los movimientos sociales que son de carácter más físico y que están relacionados con la acción, como la organización social de un colectivo. Dentro de este subapartado se encuentran, entonces, los vínculos necesarios para la integración social y la solidaridad grupal (Habermas, 1999b: 196).

La dimensión de la personalidad, en cambio, comprende la formación de la identidad personal, la adquisición de capacidades generalizadas de acción y la sintonización de las vidas individuales con formas de vida colectivas (Habermas, 1999b: 196 y 201).

Tenemos, en definitiva, unas pautas de interpretación válidas para comprender las interdependencias entre la sociología del grupo, la socialización del individuo y los procesos culturales, relacionados todos ellos con la apropiación mediática.

\subsubsection{Aplicaciones en el estudio de una nueva audiencia}

En tercer lugar, la dicotomía "mundo de vida" / "sistema" también puede ser empleada en el estudio de una nueva audiencia con un comportamiento mediático diferente al de los nativos o autóctonos españoles. Constituye un programa teórico idóneo para comprender a un público con patrones de conducta sociocultural distintos, ambientes sociales ajenos a los de la sociedad mayoritaria ${ }^{6}$, estrategias de vida transnacionales (y en algunos casos incluso un nuevo tipo de nomadismo), así como el mantenimiento de lealtades e identificaciones múltiples con las culturas y los Estados (Bauböck, 2004). Estas características también nos hablan de una nueva forma de consumo mediático, con causas motivacionales y afectivas diferentes a las del público sedentario de los Estados nación.

Con la ayuda del material conceptual habermasiano, entendemos que las minorías étnicas se encuentran delante de una sociedad que se desdobla en un Lebenswelt y en un sistema ajeno que los someten a una relación de jerarquía asimétrica. La diáspora está en situación de inferioridad desde un punto de vista del sistema porque, a grandes rasgos, la sociedad anfitriona marca las directrices administrativas, legislativas y económicas. Si al hecho de que a menudo el inmigrante se encuentra en el vértice de, al menos, dos Lebenswelt, añadimos que "los mundos de vida" de los autóctonos son hegemónicos, tenemos factores que pueden provocar dilemas para la configuración del individuo de la diáspora, para la reproducción cultural y la cohesión del tejido étnico.

En términos de Schütz, la pauta cultural de la sociedad mayoritaria no tiene para la alteridad la autoridad de un sistema verificado de recetas, porque no comparte la tradición histórica de sus ciudadanos (Schütz, 2003: 100). El individuo de una minoría étnica lee su realidad a partir de las conceptualizaciones convencionales del sentido común de su mundo de vida (Schütz, 2003: 288). Las reglas que lo guían, su historia y su estilo de vida normal se encuentran bajo la amenaza de su desaparición en la sociedad de acogida (Schütz, 2003: 106).

6.- Determinados, en alguna medida, por una comunidad étnica, cadenas migratorias y una relación intensa con las nuevas tecnologías y los medios de comunicación (Altarriba, 2008). 
Además, las fuentes de sus recursos sociales y culturales son más reducidas que las de los ciudadanos del país de acogida porque sus "mundos de vida" se han de refugiar, articular y actualizar en las comunidades étnicas, en las actividades impulsadas por la representación administrativa del país emisor de la inmigración y en la infraestructura de los medios a su alcance.

En este punto y, de nuevo, con la ayuda de Habermas, también acuñamos el concepto "comunidades de comunicación", formadas por las minorías étnicas que subsisten en diferentes grados y maneras en el seno de una sociedad mayoritaria. Estas iniciativas buscan posibilidades de expresión y comunicación propias en una sociedad que impone su "mundo de la vida" y su sistema para la configuración de la personalidad y para la cohesión social (Habermas, 1999b:560). La meta es encontrar condiciones propicias para el desarrollo de una identidad personal y colectiva diferenciadas del resto.

\section{La recepción}

Nuestra propuesta no trata de encajar, a la fuerza, la recepción en una teoría preestablecida. Es más bien un punto de partida antes que un punto de llegada, una interpretación abierta a la improvisación antes que un guión cerrado con un final previsible. En nuestra voluntad está mantener una relación libre con la recepción mediática, situándola en el centro de nuestra mirada, pero sin ocupar todo nuestro campo de visión.

Entendemos la recepción no como una instantánea fija, sino como una imagen en movimiento que forma parte de un engranaje de relaciones recíprocas con los tres grandes elementos estructurales del "mundo de la vida". Pero, ¿por qué no podemos acercarnos a este proceso en curso como si fuera un compartimento estanco o un atolón aislado, sin el efecto de sus mareas? Porque creemos que las mediaciones de las que hablamos van al quid de esta actividad y la moldean. De ahí que sea tan importante el consumo mediático en sí mismo como sus conexiones con las áreas de nuestro marco teórico, porque en la explicación de su dinamismo está su naturaleza.

En sintonía con nuestro marco teórico, el término "apropiación" refleja bien el concepto de recepción mediática porque describe su proceso completo. Uno de estos procesos va hacia fuera, trascendiendo a la persona, porque la recepción desencadena un proceso de socialización que supera el escenario inmediato del consumo mediático. En este punto nos inspiramos también en los estudios de John B. Thompson (1998: 67) quien define la recepción como una actividad que va más allá de ella misma, en un proceso de construcción narrativo alejado del contexto inicial de recepción.

Pero la recepción describe, a la vez, un camino que va hacia dentro, que incluye el procesamiento individual del contenido mediático. Creemos que los elementos del "mundo de la vida" (culturales, sociales e individuales) y del "sistema" son filtros que inciden en los patrones de interpretación del consumo y que se incorporan a la vida individual.

Sin embargo, la mayoría de trabajos se quedan varados en el primer movimiento de la recepción, su procesamiento individual, en un análisis que pasa por alto los procesos 
culturales, sociales e individuales de la realidad cotidiana que inciden en el nivel personal de la recepción. Se limitan, más bien, a la identificación del medio escogido por el receptor y al impacto que tiene el medio de comunicación, estudiando a los consumidores como miembros aislados sin sus respectivas relaciones sociales.

\section{Reflexiones finales}

En el estudio de la inmigración desde las Ciencias de la Comunicación, la recepción mediática es uno de los campos que cuenta con menos bibliografía en España. Sin embargo, la mayor parte de la producción científica se centra en los medios generalistas españoles, bien en las elecciones mediáticas de los inmigrantes, bien en la evaluación del impacto directo sobre una muestra de personas españolas. En este contexto, brilla por su ausencia la aproximación a los usos que se derivan de los medios especialmente dirigidos a la diáspora. Este artículo se inscribe en esta última tendencia, prácticamente inexistente dentro y fuera de España.

Pero además de aventurarnos en una vía inexplorada, este trabajo nace con la voluntad de romper con una orientación excesivamente instrumental del consumo mediático, que parte del sesgo y de los intereses de la sociedad de acogida. Buscamos lecturas más profundas de la recepción, más allá de los tópicos que abordan la continuidad o discontinuidad con la cultura de origen (Amezaga, 2008; Chavero y García, 2005; Mendieta, 2008) y la integración en la sociedad de acogida (Mendieta, 2008; Altarriba, 2008).

Por otro lado, con la investigación en curso pretendemos superar la institucionalización en la que suelen caer unas investigaciones que al final sólo logran apuntar tendencias y descripciones superficiales de los hábitos de consumo. Y es que gran parte de las investigaciones son cuantitativas, cuando no incursiones cualitativas de carácter descriptivo también. No se suele indagar en el porqué de las elecciones mediáticas en el marco de concepciones teóricas de fondo y de opciones metodológicas bien desarrolladas (IOÉ, 2010: 95), que eviten que los objetivos de la investigación se queden a medio camino.

Gracias a Habermas se ha delimitado el escenario en el que tiene lugar la recepción mediática, se han establecido niveles de análisis micro y macrosociales y se han hallado parámetros lo suficientemente flexibles como para estudiar a un público que desarrolla pautas socioculturales y una experiencia mediática diferentes a las del resto de la sociedad mayoritaria.

Del mismo modo, mediante este paradigma comunicativo, de profunda raíz filosófica y que tiene un modelo claro de sociedad en dos planos, damos con un concepto amplio de la recepción mediática, en la que los factores sociales, culturales e individuales están llamados a jugar un papel relevante. Sea como fuere, lo más importante aquí es tener presente que la socialización del individuo, la integración social y la reproducción cultural se ponen en juego en la recepción mediática de una forma escasamente estudiada hasta la fecha.

La relación "mundo de la vida" / "sistema" va a ayudar a contextualizar apropiaciones que tienen un carácter motivacional y afectivo especiales, permitiendo superar el plano 
descriptivo en el que se mueve la mayoría de los trabajos de recepción, para acceder a la interpretación de sus dietas mediáticas. Con esta óptica panorámica podemos interpretar, en su justa dimensión, el ciclo completo de la actividad receptora en los dos sentidos de la marcha: desde el escenario inmediato del consumo mediático hasta el proceso de socialización que desencadena.

A través del Lebenswelt, nos acercamos también a la actividad receptora desde su naturaleza dinámica y simbólica por medio de la relación de sinergias entre los componentes estructurales del mundo de la vida. Pero, además, con el sistema aportamos una visión más pragmática e instrumental de la recepción, porque la diáspora mantiene relaciones con la administración y con la economía de mercado que condicionan su supervivencia en la sociedad anfitriona. No olvidemos que se consumen medios para satisfacer necesidades concretas, como la búsqueda de trabajo, la familiarización con la sociedad de acogida y para conocer la legislación vigente.

Si en el marco teórico explicamos, desde el "mundo de la vida", el papel de la sociología del grupo, la socialización del individuo y la cultura en la recepción mediática y en la comunicación, con nuestra propuesta metodológica damos un nuevo giro de tuerca al Lebenswelt al interpretar, en términos comunicativos, la identidad, la sociedad y la cultura. Son dos caminos en sentido opuesto (marco teórico y metodología) que coinciden en un mismo punto.

Al situar la recepción en nuestro marco teórico, comprendimos que el sujeto de estudio es el producto de las tradiciones en las que ha vivido, de los grupos solidarios a los que pertenece y de los procesos de socialización en los que ha crecido (Habermas, 2008: 326). En consecuencia, en nuestra metodología, el análisis del discurso oral tendrá en cuenta que el contenido de las narraciones orales de los entrevistados se construye en los procesos de integración social, en la socialización y en la reproducción cultural. De ahí que podamos analizar los relatos de las apropiaciones mediáticas de los inmigrantes a través de los componentes del "mundo de la vida". No en vano sabemos que la estructura interna del discurso oral de los miembros de un colectivo tiene características narrativas únicas que se encuentran en su "mundo de la vida". La estructura interna de la narración guarda entonces un paralelismo extratextual con las dimensiones del Lebenswelt, por cuanto responde al contenido semántico de las tradiciones culturales, al tiempo histórico de la sucesión de generaciones y al espacio social de los grupos socialmente integrados (Habermas, 1999b: 194-196).

Al remontar los discursos orales de nuestro futuro trabajo de campo (para encontrar y tematizar los componentes estructurales del "mundo de la vida") enseñaremos, desde un plano narrativo, la construcción de la identidad, la formación y actualización de la cohesión grupal y de la cultura. Siguiendo atentamente el conjunto de las interrelaciones de los elementos del Lebenswelt, reconstruiremos los procedimientos de interpretación y el sentido que atribuyen los inmigrantes a sus exposiciones orales. Se trata de encontrar una herramienta de análisis completa de las narraciones autobiográficas, que no sea sólo lingüística, sino también social y cultural. 


\section{Fuentes consultadas}

Acebes, R. (2000). "La transformación del sujeto y los límites de la Fenomenología". Revista de Filosofia Era, 13(24).

Altarriba, M. et álii (2008). Usos i actituds dels immigrants davant dels mitjans de comunicació. Barcelona: Consell de l'Audiovisual de Catalunya (CAC).

Amezaga, J. et álii (2008). "Biladi. Usos de la televisión por satélite entre los y las inmigrantes magrebíes en Bilbao". En Revista de estudios de comunicación Zer. Extraída el 2/IX/2011 desde http://www.ehu.es/zer/zer10/10amezaga.html

Bauböck, R. (2004). "Cómo transforma la inmigración a la ciudadanía: perspectivas internacionales, multinacionales y transnacionales". En Aubarell, A. y Zapata, R. Inmigración y procesos de cambio. Europa y el Mediterráneo en el contexto global. Barcelona: Icaria.

Concepción, L. G.; Rodrigo, M. y Medina, P. (2008). "Niveles semánticos de las representaciones sociales de la inmigración subsahariana. Los sucesos de Ceuta y Melilla según $A B C$ ". Estudios sobre el Mensaje Periodístico. Universidad Complutense de Madrid, núm. 14, pp. 129-148.

Chavero, H.; García, N. (2005). "Los hábitos del consumo televisivo de una comunidad extranjera: el caso de estudiantes latinoamericanos en Barcelona". En Revista de estudios de comunicación Zer, núm. 19, pp. 191-204. Extraída el 25/VII/2011 desde http://www.ehu.es/zer/zer19/zer19_13.pdf

Concepción, L. (2009). El mundo de la vida en las apropiaciones de los medios étnicos. [Tesina doctoral no publicada]. Barcelona: Facultat de Ciències de la Comunicació Blanquerna, Universitat Ramon Llull.

Gadamer, H. (2002). Verdad y método II. Salamanca: Sígueme.

González Escudero, E. (1999). Redes sociales, comunicación y procesos de movilidad y asentamiento de los emigrantes magrebies en Alicante: 1985-1995 [Tesis doctoral no publicada]. Bellaterra: UAB. Extraída el 10/VIII/2011 desde http://www.tdx.cat/TDX-0503103-100353

Habermas, J.

_(2008). El discurso filosófico de la modernidad. Madrid: Katz Editores.

_(1999a). Teoría de la acción comunicativa, I. Racionalidad de la acción y racionalización social. Madrid: Santillana.

_(1999b). Teoría de la acción comunicativa, II. Crítica a la razón funcionalista. Madrid: Santillana.

_(1999c). La inclusión del otro. Estudios de teoría política. Barcelona: Paidós.

_(1996). Textos y contextos. Barcelona: Ariel.

_(1994a). Teoría de la acción comunicativa: complementos y estudios previos. Madrid: Cátedra.

_(1994b). Conciencia moral y acción comunicativa. Barcelona: Edicions 62.

Husserl, E. (1991). La crisis de las ciencias europeas y la fenomenología transcendental. Barcelona: Crítica. 
IDESCAT (Institut d’Estadística de Catalunya) (2010). “Evolución de la población total y extranjera. 2000-2010

Cataluña”. Extraída el 1/09/2011 desde http://www.idescat.cat/poblacioestrangera/?lang=es

IOÉ, colectivo (2010). “PPara qué sirve el grupo de discusión? Una revisión crítica del uso de técnicas grupales en los estudios sobre migraciones”. Empiria. Revista de Metodología y Ciencias Sociales, 19 (enero-junio), pp. 73-99.

Medina, A.

_(2010). Metodología de la investigación en ciencias sociales. Área de comunicación. Proyecto docente presentado para la plaza de Profesor Titular Universitario en la Facultad de Ciencias de la Comunicación Blanquerna.

_(2004). “D’una objectivitat provisional a una ètica universal”. Trípodos, núm. 16, pp. 141-153.

Mendieta, A. (2008). "Papel de los medios de comunicación y las nuevas tecnologías en el apoyo de las identidades étnicas y en la integración de las minorías en las sociedades de acogida. El caso de España". Extraída el 2/IX/2011 desde http:/www.unavarra.es/migraciones/papers3/ Comunicacion\%20Congreso\%20Navarra\%20Ana\%20Mendieta.pdf

Moragas, M. (1993). Sociología de la comunicación de masas. Barcelona: Gustavo Gili.

Radl, R. (1998). "La teoría del actuar comunicativo de Jürgen Habermas: un marco para el análisis de las condiciones socializadoras en las sociedades modernas”. Papers, núm. 56.

Rizo, M. (2004). Prácticas culturales y redefinición de las identidades de los inmigrantes en El Raval (Barcelona): aportaciones desde la comunicación [Tesis doctoral no publicada]. Bellaterra: UAB.

Rodríguez Salazar, T. (1996). "El itinerario del concepto de mundo de la vida. De la fenomenología a la teoría de la acción comunicativa”. En Comunicación y Sociedad, núm. 27, pp. 199-214. Extraída el 10/VIII/2011 desde http://ccdoc.iteso.mx//cat.aspx?cmn=browse\&id=3331

Schütz, A. (2003). Estudios sobre teoría social. Escritos II. Buenos Aires: Amorrortu.

Thompson, J. B. (1998). Los media y la modernidad. Una teoría de los medios de comunicación. Barcelona: Paidós.

Vasileva, K. (2010). "Foreigners living in the EU are diverse and largely younger than the nationals of the EU Member States". En EUROSTAT. Statistics in focus, núm. 45. Extraída el VIII/2011 desde http://epp.eurostat.ec.europa.eu/cache/ITY_OFFPUB/KS-SF-10-045/EN/KS-SF-10-045-EN.PDF

Weber, M. (1998). La ética protestante y el espíritu del capitalismo. Barcelona: Península.

Wolf, M. (1987). La investigación de la comunicación de masas. Críticas y perspectivas. Barcelona: Paidós. 